\title{
Adapting Planck's route to investigate the thermodynamics of the spin-half pyrochlore Heisenberg antiferromagnet
}

\author{
Oleg Derzhko $\odot,{ }^{1,2}$ Taras Hutak $\odot,{ }^{1}$ Taras Krokhmalskii, ${ }^{1}$ Jürgen Schnack, ${ }^{3}$ and Johannes Richter ${ }^{4,2}$ \\ ${ }^{1}$ Institute for Condensed Matter Physics, National Academy of Sciences of Ukraine, Svientsitskii Street 1, 79011 L'viv, Ukraine \\ ${ }^{2}$ Max-Planck-Institut für Physik komplexer Systeme, Nöthnitzer Straße 38, 01187 Dresden, Germany \\ ${ }^{3}$ Fakultät für Physik, Universität Bielefeld, Postfach 100131, 33501 Bielefeld, Germany \\ ${ }^{4}$ Institut für Physik, Otto-von-Guericke-Universität Magdeburg, P.O. Box 4120, 39016 Magdeburg, Germany
}

(Received 25 February 2020; revised manuscript received 9 April 2020; accepted 28 April 2020; published 18 May 2020)

\begin{abstract}
The spin-half pyrochlore Heisenberg antiferromagnet (PHAF) is one of the most challenging problems in the field of highly frustrated quantum magnetism. Stimulated by the seminal paper of M. Planck [M. Planck, Verhandl. Dtsch. phys. Ges. 2, 202 (1900)] we calculate thermodynamic properties of this model by interpolating between the low- and high-temperature behavior. For that we follow ideas developed in detail by B. Bernu and G. Misguich and use for the interpolation the entropy exploiting sum rules [the "entropy method" (EM)]. We complement the EM results for the specific heat, the entropy, and the susceptibility by corresponding results obtained by the finite-temperature Lanczos method (FTLM) for a finite lattice of $N=32$ sites as well as by the high-temperature expansion (HTE) data. We find that due to pronounced finite-size effects the FTLM data for $N=32$ are not representative for the infinite system below $T \approx 0.7$. A similar restriction to $T \gtrsim 0.7$ holds for the HTE designed for the infinite PHAF. By contrast, the EM provides reliable data for the whole temperature region for the infinite PHAF. We find evidence for a gapless spectrum leading to a power-law behavior of the specific heat at low $T$ and for a single maximum in $c(T)$ at $T \approx 0.25$. For the susceptibility $\chi(T)$ we find indications of a monotonous increase of $\chi$ upon decreasing of $T$ reaching $\chi_{0} \approx 0.1$ at $T=0$. Moreover, the EM allows us to estimate the ground-state energy to $e_{0} \approx-0.52$.
\end{abstract}

DOI: 10.1103/PhysRevB.101.174426

\section{INTRODUCTION}

A paradigmatic highly frustrated spin model is the pyrochlore Heisenberg antiferromagnet (PHAF). The pyrochlore lattice is built of corner-sharing tetrahedra, see Fig. 1, below. There are several compounds where the magnetic atoms reside on the sites of the pyrochlore lattice and the exchange interaction is antiferromagnetic, see, e.g., Refs. [1-3].

Already the classical PHAF (i.e., for spin $S \rightarrow \infty$ ) exhibits interesting properties and its study is far from being trivial [4-10]. Thus, the ground-state manifold is highly degenerate; the model exhibits strong short-range correlations, but it does not exhibit any long-range order, and, because of the huge degeneracy of the ground state, the model is very susceptible to various perturbations.

The quantum spin $S=1 / 2$ PHAF is even more complicated. Thus, so far no accurate values for the ground-state energy $e_{0}$ for this model are available. On one hand, the $S=1 / 2$ case opens the route to new quantum phases [11]. On

Published by the American Physical Society under the terms of the Creative Commons Attribution 4.0 International license. Further distribution of this work must maintain attribution to the author(s) and the published article's title, journal citation, and DOI. Funded by the Max Planck Society. the other hand, such powerful straightforward numerical tools like standard quantum Monte Carlo or molecular dynamics simulations are not applicable for the $S=1 / 2$ PHAF. Moreover, several approximation methods developed for one- and two-dimensional quantum spin systems (e.g., density matrix renormalization group and tensor network methods) are very limited in three dimensions.

Theoretical studies of the quantum PHAF are mostly focused on ground-state properties, see, e.g., Refs. [11-25], whereas much less attention has been paid to its finitetemperature properties. One reason for that is the lack of methods to study thermodynamics of three-dimensional frustrated quantum spin systems. Among the few papers studying the thermodynamics of the $S=1 / 2$ PHAF we mention bold diagrammatic Monte Carlo simulations (stochastic sampling of all skeleton Feynman diagrams) down to the temperature $J / 6$ [26]. This paper reports data for the susceptibility $\chi(T)$ but no data for the specific heat $c(T)$. We will refer to these data for $\chi(T)$ in Sec. IV B. A comprehensive analysis of the spin- $S J_{1}-J_{2}$ Heisenberg model by employing the pseudofermion functional renormalization group technique was presented in Ref. [11]. However, this paper does not contain data for $\chi(T)$ and $c(T)$. Finally, we mention the high-temperature expansion study and the rotation-invariant Green's function study of the $S=1 / 2$ PHAF [25,27]. In these recent papers $[11,25,26]$ no evidence for a finite-temperature phase transition was found, i.e., the spin-half PHAF is most 


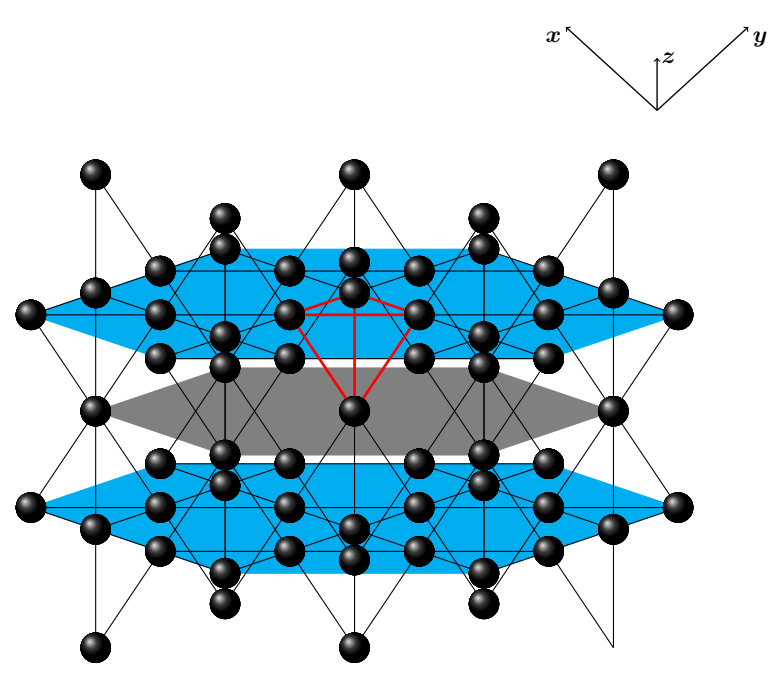

FIG. 1. The pyrochlore lattice visualized here as a threedimensional structure which consists of alternating kagome (cyan) and triangular (gray) planar layers. The four-site unit cell is marked with the red bonds.

likely a three-dimensional spin system without singularities in the specific heat and the susceptibility.

The goal of the present paper is to study the thermodynamics of the $S=1 / 2$ PHAF for the whole temperature region focussing on the specific heat $c(T)$ and the static uniform susceptibility $\chi(T)$, both being basic and easily accessible quantities in experimental studies of PHAF compounds. To deal with the above mentioned challenges when studying the finite-temperature properties of the $S=1 / 2$ PHAF, we follow M. Planck's ideas of his seminal paper in 1900 [28], see also Appendix A, and perform a sophisticated interpolation between the low- and high-temperature behavior of a thermodynamic potential, namely, the entropy $s$ as a function of internal energy $e$. For that we exploit also sum rules valid for the specific heat as proposed by B. Bernu and G. Misguich $[29,30]$, for details see Sec. III A. In what follows we call this approach the entropy method (EM). We complement our studies based on the EM by using the finite-temperature Lanczos method (FTLM) for a finite pyrochlore lattice of $N=32$ sites and the high-temperature expansion (HTE) up to order 13. In the present paper, we estimate the groundstate energy to $e_{0} \approx-0.52$ and find evidence for a gapless spectrum, i.e., for a power-law behavior of the specific heat at low temperatures and for a single maximum in $c(T)$ at about $25 \%$ of the exchange coupling.

The paper is organized as follows. We begin with introducing the model (Sec. II) and the description of the exploited methods (Sec. III). We report our findings obtained by the FTLM (finite lattices) and by the HTE and EM (infinite lattice) in Sec. IV. We summarize our results in Sec. V.

\section{MODEL}

We consider the Heisenberg model on the pyrochlore lattice (see Fig. 1) given by the Hamiltonian

$$
H=\sum_{\langle m \alpha, n \beta\rangle} \boldsymbol{S}_{m \alpha} \cdot \boldsymbol{S}_{n \beta} .
$$

We have set the antiferromagnetic nearest-neighbor coupling to unity, $J=1$, fixing the energy scale. The sum in Eq. (2.1) runs over all nearest-neighbor bonds and $S_{m \alpha}^{2}=3 / 4$.

The pyrochlore lattice consists of four interpenetrating face-centered-cubic sublattices. The origins of these four sublattices are located at $\mathbf{r}_{1}=(0,0,0), \mathbf{r}_{2}=(0,1 / 4,1 / 4)$, $\mathbf{r}_{3}=(1 / 4,0,1 / 4)$, and $\mathbf{r}_{4}=(1 / 4,1 / 4,0)$. The sites of the face-centered-cubic lattice are determined by $\mathbf{R}_{m}=m_{1} \mathbf{e}_{1}+$ $m_{2} \mathbf{e}_{2}+m_{3} \mathbf{e}_{3}$, where $m_{1}, m_{2}, m_{3}$ are integers and $\mathbf{e}_{1}=$ $(0,1 / 2,1 / 2), \mathbf{e}_{2}=(1 / 2,0,1 / 2), \mathbf{e}_{3}=(1 / 2,1 / 2,0)$. As a result, the $N$ pyrochlore lattice sites are labeled by $m \alpha, \mathbf{R}_{m \alpha}=$ $\mathbf{R}_{m}+\mathbf{r}_{\alpha}$, where $m=1, \ldots, \mathcal{N}, \mathcal{N}=N / 4$ is the number of unit cells, and $\alpha=1,2,3,4$ labels the sites in the unit cell.

There are a few compounds with magnetic atoms residing on pyrochlore-lattice sites with antiferromagnetic nearestneighbor exchange interactions, which can be considered as experimental realizations of the quantum PHAF. First of all we may mention two magnetically frustrated molybdate pyrochlores: $S=1 / 2$ oxynitride $\mathrm{Lu}_{2} \mathrm{Mo}_{2} \mathrm{O}_{5} \mathrm{~N}_{2}$ and $S=1$ oxide $\mathrm{Lu}_{2} \mathrm{Mo}_{2} \mathrm{O}_{7}$ [31,32]. Besides the fluoride $\mathrm{NaCaNi}_{2} \mathrm{~F}_{7}$ which provides a good realization of the $S=1$ PHAF [33,34], we may mention the molybdate $\mathrm{Y}_{2} \mathrm{Mo}_{2} \mathrm{O}_{7}$ [35-37], the chromites $A \mathrm{Cr}_{2} \mathrm{O}_{4}(A=\mathrm{Mg}, \mathrm{Zn}, \mathrm{Cd})$ [38-40], or $\mathrm{FeF}_{3}$ [41].

\section{METHODS}

\section{A. Entropy method (EM)}

In accordance with M. Planck's strategy to derive the energy distribution of the black-body radiation [28,42], the EM is an interpolation scheme that combines presumed knowledge on high- and low-temperature properties and, in addition, exploits sum rules for the specific heat $c(T)$ in a clever way. The EM as used in the present paper was introduced in 2001 by B. Bernu and G. Misguich [29]. The method has been afterwards used, modified, and extended in Refs. [30,43-46]. Below we explain briefly this procedure for self consistency.

Within the framework of the EM, we use the microcanonical ensemble working with the entropy per site $s$ as a function of the energy per site $e, s(e)$, in the whole (finite) range of energies. The temperature $T$ and the specific heat per site $c$ are given by the formulas

$$
T=\frac{1}{s^{\prime}}, \quad c=-\frac{s^{\prime 2}}{s^{\prime \prime}},
$$

where the prime denotes the derivative with respect to $e$. These equations form a parametric representation of the dependence $c(T)$. Knowing the high-temperature series for $c(T)$ up to $n$th order, $c(T)=\sum_{i=2}^{n} d_{i} \beta^{i}+\mathcal{O}\left(\beta^{n+1}\right)\left(d_{1}=0\right), \beta=1 / T$, we immediately get the series for $s(e)$ around the maximal energy $e_{\infty}=0$ up to the same order $n$,

$$
\left.s(e)\right|_{e \rightarrow e_{\infty}=0} \rightarrow \ln 2+\sum_{i=2}^{n} a_{i} e^{i},
$$

where the coefficients $a_{i}$ are known functions of the coefficients $d_{i}$, see Appendix A of Ref. [29]. The behavior of $s(e)$ as $e$ approaches the (minimal) ground-state energy $e_{0}$ (i.e., as the temperature approaches 0 ) is also supposed to be known. 
It is

$$
\left.s(e)\right|_{e \rightarrow e_{0}} \propto\left(e-e_{0}\right)^{\frac{\alpha}{1+\alpha}}
$$

if $c(T)$ vanishes as $T^{\alpha}$ when $T \rightarrow 0$ (gapless excitations) and

$$
\left.s(e)\right|_{e \rightarrow e_{0}} \propto-\frac{e-e_{0}}{\Delta}\left(\ln \left[\Delta\left(e-e_{0}\right)\right]-1\right)
$$

if $c(T)$ vanishes as $T^{-\alpha} \exp (-\Delta / T), \alpha=2$, when $T \rightarrow 0$ (gapped excitations). Therefore we proceed differently in the gapless case and in the gapped case. Here it is assumed that $e_{0}$ and $\alpha$ are known (gapless case) or $e_{0}$ is known and $\alpha=2$ (gapped case).

In the gapless case, we introduce the auxiliary function [30]

$$
G(e)=\frac{(s(e))^{\frac{1+\alpha}{\alpha}}}{e-e_{0}}
$$

and approximate it as

$$
G_{\text {app }}(e)=G(0)[u, d](e), \quad G(0)=\frac{(\ln 2)^{\frac{1+\alpha}{\alpha}}}{-e_{0}} .
$$

Here $[u, d](e)=P_{u}(e) / Q_{d}(e)$ is a Padé approximant, where the coefficients of the polynomials $P_{u}(e)$ and $Q_{d}(e)$ (of order $u$ and $d$, respectively, $u+d \leqslant n$ ) are determined by the condition that the expansion of $[u, d](e)$ has to agree with the power series of $G(e) / G(0)$ [which follows from Eqs. (3.5) and (3.2)] up to order $\mathcal{O}\left(e^{u+d}\right)$. Of course, $G(0)=G_{\text {app }}(0)$. The approximate entropy follows by inverting Eq. (3.5)

$$
s_{\text {app }}(e)=\left[\left(e-e_{0}\right) G_{\text {app }}(e)\right]^{\frac{\alpha}{1+\alpha}} .
$$

The prefactor $A$ in the power-law decay of the specific heat $c(T)$ for $T \rightarrow 0, c(T) \rightarrow A T^{\alpha}$, is given by

$$
A_{\text {app }}=\frac{\alpha^{1+\alpha}}{(1+\alpha)^{\alpha}}\left[G_{\text {app }}\left(e_{0}\right)\right]^{\alpha} .
$$

In the gapped case, we introduce the auxiliary function [29]

$$
G(e)=\left(e-e_{0}\right)\left(\frac{s(e)}{e-e_{0}}\right)^{\prime}
$$

and approximate it as

$$
G_{\text {app }}(e)=G(0)[u, d](e), \quad G(0)=\frac{\ln 2}{e_{0}} .
$$

Here $[u, d](e)=P_{u}(e) / Q_{d}(e)$ again is a Padé approximant, where the coefficients of the polynomials $P_{u}(e)$ and $Q_{d}(e)$ (of order $u$ and $d$, respectively, $u+d \leqslant n$ ) are determined by the condition that the expansion of $[u, d](e)$ has to agree with the power series of $G(e) / G(0)$ [which follows now from Eqs. (3.9) and (3.2)] up to order $\mathcal{O}\left(e^{u+d}\right)$. Of course, $G(0)=G_{\text {app }}(0)$. The approximate entropy follows by inverting Eq. (3.9)

$$
\frac{s_{\text {app }}(e)}{e-e_{0}}=\frac{\ln 2}{-e_{0}}-\int_{e_{0} \leqslant e \leqslant 0}^{0} \mathrm{~d} \xi \frac{G_{\text {app }}(\xi)}{\xi-e_{0}} .
$$

From the technical point of view, before performing the integration in the right-hand side of Eq. (3.11) one may perform the partial fraction expansion of the integrand which is obviously a rational function. The excitation gap $\Delta$ in the decay of the specific heat $c(T)$ for $T \rightarrow 0, c(T) \propto T^{-2} \exp (-\Delta / T)$, is given by

$$
\Delta_{\text {app }}=-\frac{1}{G_{\text {app }}\left(e_{0}\right)} .
$$

Until now we considered the EM for zero magnetic field $h=0$. Of course, for nonzero $h$ the thermodynamic functions depend on $h$, i.e., the entropy is now $s(e, h)$. The magnetization per site $m$ and the uniform susceptibility per site $\chi$ are given by the formulas [46]

$$
m=\frac{1}{(s(e, h))^{\prime}} \frac{\partial s(e, h)}{\partial h}, \quad \chi=\frac{m}{h},
$$

where the last equation implies that $h$ is infinitesimally small. Clearly, the HTE coefficients for the specific heat are also changed. Simple algebra yields

$$
d_{i} \rightarrow d_{i}+\frac{(i-1) i}{2} c_{i-1} h^{2}, \quad i=2, \ldots, n
$$

we use here the high-temperature series for the static uniform susceptibility $\chi(T)=\sum_{i=1}^{n} c_{i} \beta^{i}+\mathcal{O}\left(\beta^{n+1}\right), \beta=1 / T$. The expression (3.2) for the series of $s$ is valid, however, the coefficients $a_{i}$ are now known functions of the coefficients $d_{i}$, $c_{i}$, and $h$. For the gapless case all reasonings in Eqs. (3.3), (3.5) to (3.8) hold with the only difference that the ground-state energy now is $e_{0}-\chi_{0} h^{2} / 2$, where $\chi_{0} \equiv \chi(T=0)$ is the ground-state susceptibility which is assumed to be known. The approximate entropy in Eq. (3.7) now also depends on $h$, i.e., $s_{\text {app }}(e, h)$. For the case of gapped magnetic excitations the ground-state energy remains unchanged, because $\chi_{0}=0$, and therefore all the Eqs. (3.4), (3.9) to (3.12) are valid. Again, the approximate entropy in Eq. (3.11) now also depends on $h$, i.e., $s_{\text {app }}(e, h)$.

In summary, knowing the high-temperature series of $c(T)$ and $\chi(T)$ together with (i) the ground-state energy $e_{0}$, the exponent $\alpha$, and the ground-state susceptibility $\chi_{0}$ for the gapless case or (ii) only the ground-state energy $e_{0}$ for the gapped case, we obtain $c(T)$ and $\chi(T)$ at all temperatures. For that, we use $s_{\text {app }}(e, h)$ which yields the specific heat $c(T)$ by Eq. (3.1) and the susceptibility $\chi(T)$ by Eqs. (3.13) and (3.1).

Based on previous experience with the EM [29,30,4346], we use the following strategy: We discard those Padé approximants in $G_{\text {app }}(e)$, Eqs. (3.6) and (3.10), which give unphysical solutions; the remaining ones are called "physical." Moreover, we focus on those input parameter sets for which interpolations based on different Padé approximants lead to data sets for $c(T)$ and $\chi(T)$ being quite close to each other. For further details about the EM in the context of the $S=1 / 2$ PHAF see Sec. IV B.

\section{B. Finite-temperature Lanczos method (FTLM)}

The FTLM is an efficient and very accurate approximation to calculate thermodynamic quantities of quantum spin systems on finite lattices of $N$ sites at arbitrary temperatures. It is an unbiased numerical approach, where thermodynamic quantities such as the specific heat and the susceptibility are determined using trace estimators [47-55]. The key element is the approximation of the partition function $Z$ using a Monte- 
Carlo like representation of $Z$, i.e., the sum over a complete set of $2^{N}$ basis vectors present in $Z$ is replaced by a much smaller sum over $R$ random vectors $|v\rangle$ for each subspace $\mathcal{H}(\gamma)$ of the Hilbert space, where except the conservation of total $S^{z}$ we also use the lattice symmetries of the Hamiltonian to decompose the full Hilbert space into mutually orthogonal subspaces labeled by $\gamma$. The exponential of the Hamiltonian is then approximated by its spectral representation in a Krylov space spanned by the $N_{L}$ Lanczos vectors starting from the respective random vector $|v\rangle$. The FTLM representation of the partition function finally reads

$$
Z(T) \approx \sum_{\gamma=1}^{\Gamma} \frac{\operatorname{dim}(\mathcal{H}(\gamma))}{R} \sum_{\nu=1}^{R} \sum_{n=1}^{N_{L}} \exp \left(-\frac{\epsilon_{n}^{(\nu)}}{T}\right)|\langle n(\nu) \mid \nu\rangle|^{2},
$$

where $|n(v)\rangle$ is the $n$th eigenvector of $H$ in the Krylov space with the corresponding energy $\epsilon_{n}^{(v)}$. To perform the symmetry-decomposed numerical Lanczos calculations we use J. Schulenburg's SPINPACK code [56,57].

\section{High-temperature expansion (HTE)}

The HTE is a universal approach to discuss the thermodynamics of spin systems [58]. In the present study we use the Magdeburg HTE code developed mainly by A. Lohmann $[27,59,60]$ (which is freely available at [61]) in an extended version up to 13th order, see Appendix B. With this tool, we compute the series of the specific heat $c(T)=$ $\sum_{i=2}^{n} d_{i} \beta^{i}+\mathcal{O}\left(\beta^{n+1}\right)\left(d_{1}=0\right)$ and the static uniform susceptibility $\chi(T)=\sum_{i=1}^{n} c_{i} \beta^{i}+\mathcal{O}\left(\beta^{n+1}\right)$ with respect to the inverse temperature $\beta=1 / T$.

To extend the region of validity of the "raw" HTE series we may use Padé approximants $[m, n]=P_{m}(\beta) / Q_{n}(\beta)$, where $P_{m}(\beta)$ and $Q_{n}(\beta)$ are polynomials in $\beta$ of order $m$ and $n$, respectively. The coefficients of the polynomials $P_{m}(\beta)$ and $Q_{n}(\beta)$ are determined by the condition that the expansion of $[m, n]$ has to agree with the initial power series up to order $\mathcal{O}\left(\beta^{m+n}\right)$.

\section{RESULTS}

\section{A. Finite lattices}

In Ref. [24] finite lattices of $N=28$ and $N=36$ sites are used to discuss ground-state properties. These lattices are built by stacked alternating triangular and kagome layers imposing periodic boundary conditions within the layers but open boundary conditions perpendicular to them. We have calculated the HTE series for these finite lattices. We compare these finite-lattice series with the corresponding HTE series of the infinite pyrochlore lattice to judge the finite lattices. We found that for $c(T)$ all HTE coefficients are different. For the susceptibility $\chi(T)$ only the lowest-order term coincides, i.e., the agreement is only marginally better. This drastic difference between the finite lattices and the infinite pyrochlore lattice can be attributed to the edge spins stemming from the imposed open boundary conditions. Thus, we conclude that the finite lattices of $N=28$ and $N=36$ used in Ref. [24] are not appropriate to discuss the thermodynamics of the PHAF. However, we note that they can be useful to discuss
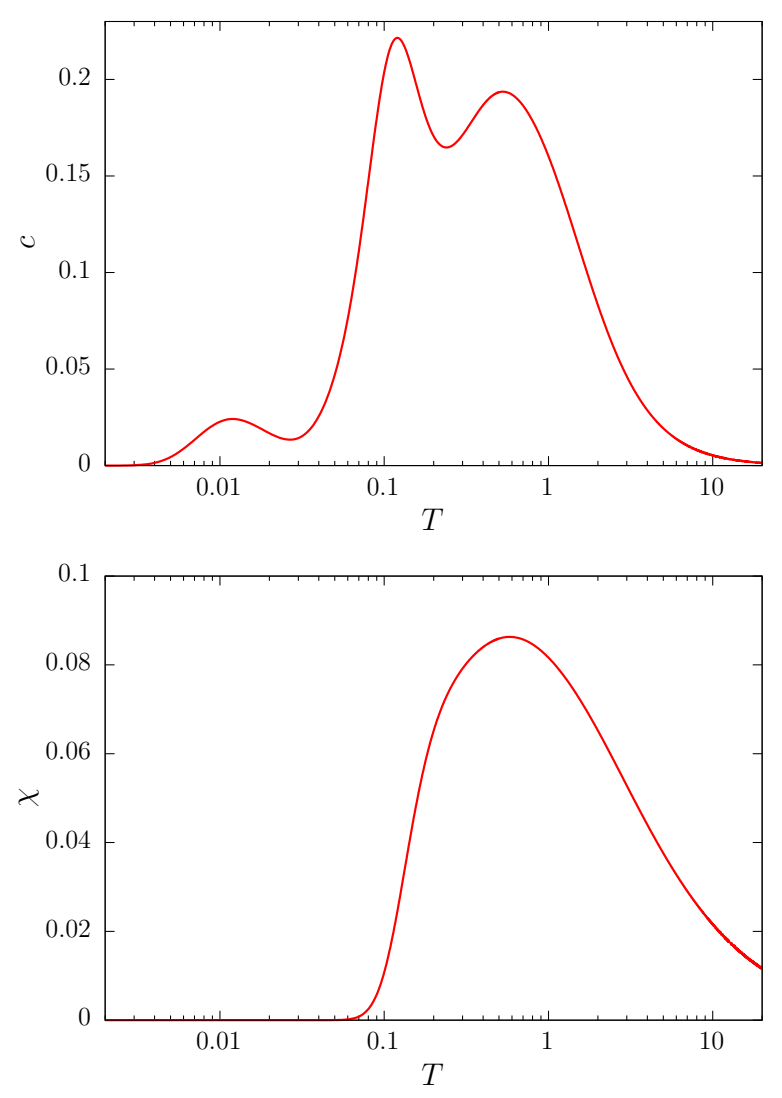

FIG. 2. FTLM data $(R=100)$ for the temperature dependence (logarithmic scale) of (top) the specific heat per site $c(T)$ and (bottom) the static uniform susceptibility per site $\chi(T)$ of the PHAF of $N=32$ sites.

the ground-state properties, e.g., spin-spin correlations when considering spins away from the edge spins.

A more suitable finite lattice is the one with $N=32$ sites imposing periodic boundary conditions in all directions. This lattice contains eight face-centered-cubic cells, i.e., the edge vectors go along the face-centered-cubic basis vectors and have twice the length of these. For this lattice the HTE series for $c(\chi)$ coincides up to third (fourth) order with that of the infinite lattice.

The FTLM is the adequate approach to study the finite $S=1 / 2$ PHAF of $N=32$ sites. In Fig. 2 we show data for $c(T)$ (top) and $\chi(T)$ (bottom) over a wide temperature range using a logarithmic $T$ scale. The specific heat exhibits the typical main maximum at $T=0.53$ and, in addition, two low- $T$ maxima at $T=0.012$ and at $T=0.117$. While the maximum at $T=0.012$ is certainly a finite-size effect, one can speculate that the other low- $T$ maximum at $T=0.117$ signals an extra low-energy scale set by low-lying singlets (see the density of states shown in the inset of the middle panel of Fig. 3) that might be also relevant for the infinite system. Such a feature has been observed in low-dimensional highly frustrated quantum magnets, e.g., the spin-half kagome Heisenberg antiferromagnet (HAF), where the existence of such an extra low- $T$ peak is a subject of a long-standing and ongoing debate [30,54,62-67]. However, in the threedimensional PHAF the finite-size effects are undoubtedly 

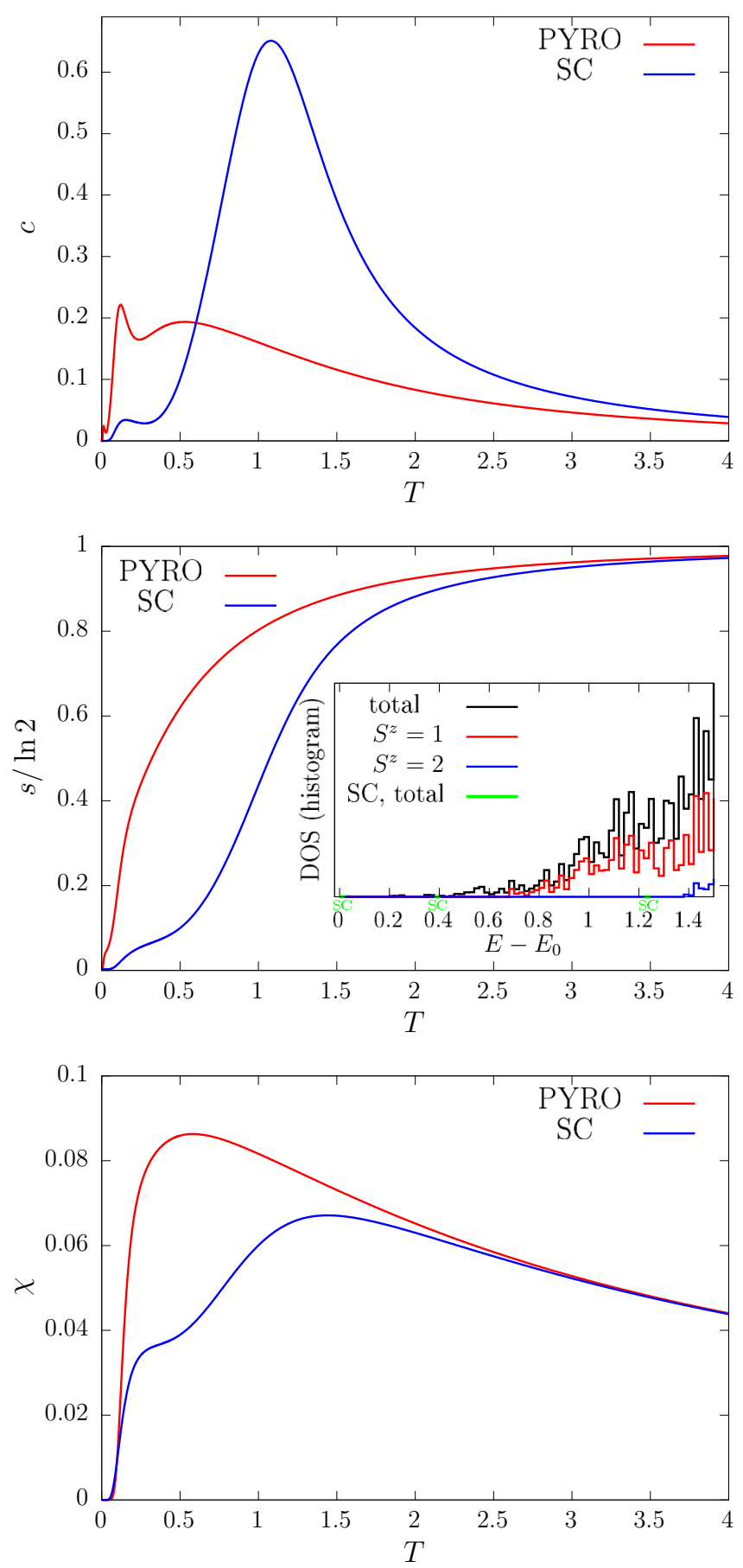

FIG. 3. Comparison of FTLM data $(R=100)$ of the PHAF of $N=32$ sites with corresponding ones of the simple-cubic HAF of $N=32$ sites. (Top) Specific heat per site $c(T)$. (Middle) Entropy per site $s(T)$. (Bottom) Static uniform susceptibility per site $\chi(T)$. The inset in the middle panel shows the histogram low-energy density of states (arbitrary units). Note that for the simple-cubic HAF there are only three tiny bars in the energy region shown here (their positions are indicated by green labels "sc").

stronger than in the two-dimensional kagome HAF. Thus, to conclude a double-peak structure in $c(T)$ from our FTLM data is inappropriate. For the static uniform susceptibility $\chi(T)$ the low-lying singlets are not relevant and $\chi(T)$ does not show extra peaks except the well-pronounced maximum that is typ-

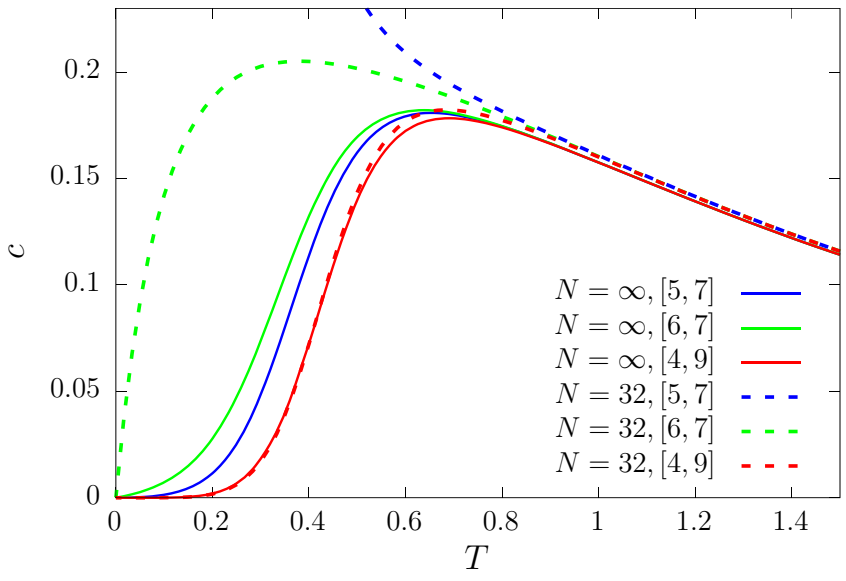

FIG. 4. Several Padé approximants of the specific heat $c(T)$ of the PHAF: comparison of the finite lattice of $N=32$ (broken) with the infinite lattice (solid).

ical for finite spin systems with $\chi_{0} \equiv \chi(T=0)=0$. Again, this behavior might be not representative for the infinite system, particularly, in case that $\chi_{0}>0$ for $N \rightarrow \infty$.

To quantify the temperature region where the finite $N=$ 32 lattice may be representative for the infinite lattice we compare in Figs. 4 and 5 several Padé approximants of the HTE series of the finite and the infinite lattices. Obviously, the data for $N=32$ and $N=\infty$ coincide only down to $T \sim 0.7$, thus, indicating that finite pyrochlore lattices accessible by FTLM are not suitable to discuss the thermodynamics of the spin-half PHAF below this temperature.

Nevertheless, the finite-size data for the PHAF are useful to demonstrate the frustration effects. Although the suppression of energy scales in frustrated magnets is a known fact nowadays, see, e.g., Refs. [54,68], the discussion of the specific case of the $S=1 / 2$ PHAF might be instructive. For that we may compare the PHAF with the $S=1 / 2$ HAF on the simplecubic lattice, because both have six nearest neighbors, i.e., a simple mean-field decoupling of the Heisenberg Hamiltonian would yield identical thermodynamics. However, the simplecubic HAF exhibits a finite-temperature phase transition to

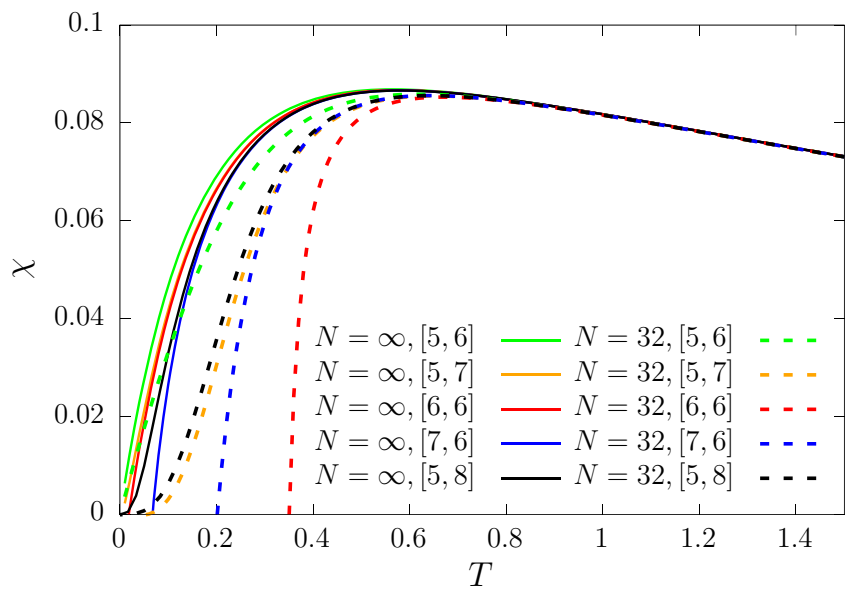

FIG. 5. Several Padé approximants of the uniform susceptibility $\chi(T)$ of the PHAF: comparison of the finite lattice of $N=32$ (broken) with the infinite lattice (solid). 
Néel order, but the PHAF does not order. Thus, we compare both models on finite lattices of $N=32$ sites, where the simple-cubic finite-temperature phase transition is irrelevant, see Fig. 3, where we compare FTLM data of the specific heat, the entropy, and the susceptibility using a linear $T$ scale for $N=32$. The tremendous influence of frustration is visible at all temperature scales. In particular, the spectrum at low energies in the frustrated system is much denser than that of the unfrustrated one [see the density of states (histogram, $\Delta E=0.02$ ) in the inset in the middle panel of Fig. 3], thus leading to the drastic differences at low $T$, see the upper and middle panels of Fig. 3. Remarkably, the noticeable differences in all quantities are present at pretty high temperatures. Only, beyond $T \gtrsim 3$ the corresponding curves approach each other. A striking effect of frustration is also the shift of the maximum in $\chi(T)$ to lower temperatures, see the lower panel of Fig. 3.

\section{B. Infinite lattice}

Let us now move to a detailed investigation of the infinite PHAF by using the EM interpolation scheme, see Sec. III A. Using our Magdeburg HTE code $[27,59,60]$ we have created the HTE series for $c(T)$ and $\chi(T)$ up to order 13 (see Appendix B) that provides the high-temperature input for the EM. Since the EM finally uses Padé approximants of a power series of $s(e)$ derived from the initial HTE series, the HTE input determines the highest order of the Padé approximants of the EM interpolation scheme.

As a low-temperature input for the EM we need the ground-state energy $e_{0}$. There is a large variety of reported values for $e_{0}$ of the spin-half PHAF ranging from $e_{0}=-0.57$ to $e_{0}=-0.45$, namely, $e_{0}=-0.572$ [69], -0.56 [15], -0.49 [12,16], -0.482081 [24], -0.466971 [24], -0.459 [22], -0.457804 [13], -0.45093 [25], -0.4473 [23], i.e., accurate values for $e_{0}$ are missing. We also need the low-temperature law for $c(T)$, where we have to distinguish between gapped and gapless behavior (cf. Sec. III A). Finally, in case of gapless excitations the exponent $\alpha$ of the power law is an input parameter, and for the susceptibility $\chi_{0} \equiv \chi(T=$ 0 ) is required as input. Fortunately, for gapped excitations the value of the gap is not needed as an input, it is rather an output of the EM. Moreover, we have $\chi_{0} \equiv \chi(T=0)=0$ in this case.

We begin with the specific heat $c(T)$ for which the EM is better justified (two sum rules are exploited). Actually, the low-temperature law for $c(T)$ is not known for the $S=1 / 2$ PHAF. Advantageously, the previous ample of experience with the EM [29,30,43-46] provides valuable hints to overcome this difficulty. Thus, in case that these input data are too far from the true (but possibly unknown) data one gets inconsistent or unphysical results. Hence, to get physical (i.e., pole free) Padé approximants requires reasonable values for $e_{0}$ and reasonable assumptions on the low- $T$ behavior of $c(T)$. Moreover, getting a large number $n_{\mathrm{P}}$ of similar Padé approximants for a certain input data set indicates the physical relevance of this set. On the other hand, the appearance of significant differences between the various Padé approximants is a criterion to discard the corresponding input set. The successfulness of this strategy has been demonstrated for several examples

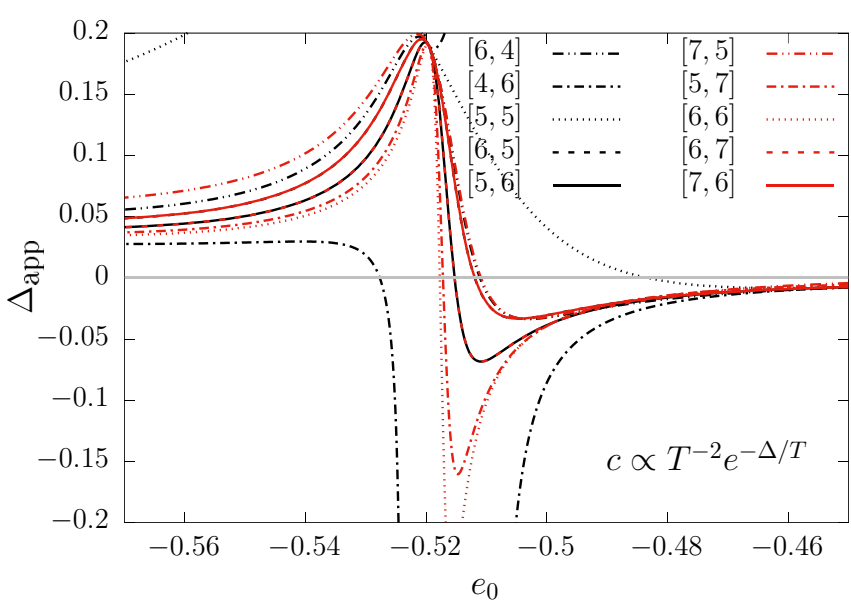

FIG. 6. EM for gapped spectrum: The gap $\Delta_{\text {app }}$ as it follows from Eq. (3.12) versus the ground-state energy $e_{0}$ varied in region $-0.57 \ldots-0.45$.

where excellent reference data are available, in particular, for the $S=1 / 2 X Y$, HAF, and Ising chains [29,44], for the $S=1$ HAF (Haldane) chain [29], for the $S=1 / 2$ square-lattice and triangular-lattice Heisenberg ferro- and antiferromagnets [29] or for the $S=1 / 2$ kagome-lattice HAFs [30,44,46]. In what follows, we often show bundles of curves which represent different Padé approximants. The closeness of the various curves (due to which they sometimes are not very well distinguishable) serves as a qualitative indication of the precision of the EM estimates and in particular of their independence of the precise values of the chosen parameters, see Ref. [30].

By combining different assumptions on the ground-state energy and low- $T$ behavior of $c(T)$ of the PHAF we have generated a large set of temperature profiles for the specific heat. In the next step, we use the guidelines described above to evaluate the used input data, this way obtaining definite conclusions on their relevance. In sum, a crucial criterion is that a particular input data set leads to a close bundle of temperature profiles obtained by many physical Padé approximants. Since the high-temperature part is per se identical this criterion concerns the temperature region $T \lesssim 0.5$. In what follows, we will present in the main text only data for the most relevant input sets, whereas presentation of some other illustrative results, only briefly mentioned in the main text, are transferred to Supplemental Material [70].

As a first result, we found that the assumption of gapped excitations is not favorable for the following reasons. We varied $e_{0}$ in the region $-0.57 \ldots-0.45$ and calculated the gap given in Eq. (3.12) using different Padé approximants, where we focused on nearly diagonal Padé approximants $[u, d](e)$, $u \sim d$, constructed from HTE data of 10th, 11th, 12th, and 13th order, see Fig. 6. We find that the gap $\Delta_{\text {app }}$, Eq. (3.12), is negative if the ground-state energy exceeds approximately -0.515 , thus providing evidence that a gapped spectrum together with $e_{0} \gtrsim-0.515$ can be excluded. For ground-state energies in the region $-0.519 \ldots-0.517$ we obtain $\Delta_{\text {app }}=$ $0.16 \ldots 0.18$, see Fig. 6 (and Fig. 1 in Ref. [70]), and there is a decent number $n_{\mathrm{P}}$ of Padé approximants yielding similar $c(T)$ profiles, see Fig. 7. For $e_{0}$ less than -0.521 the number of physical Padé approximants noticeably decreases. Although 


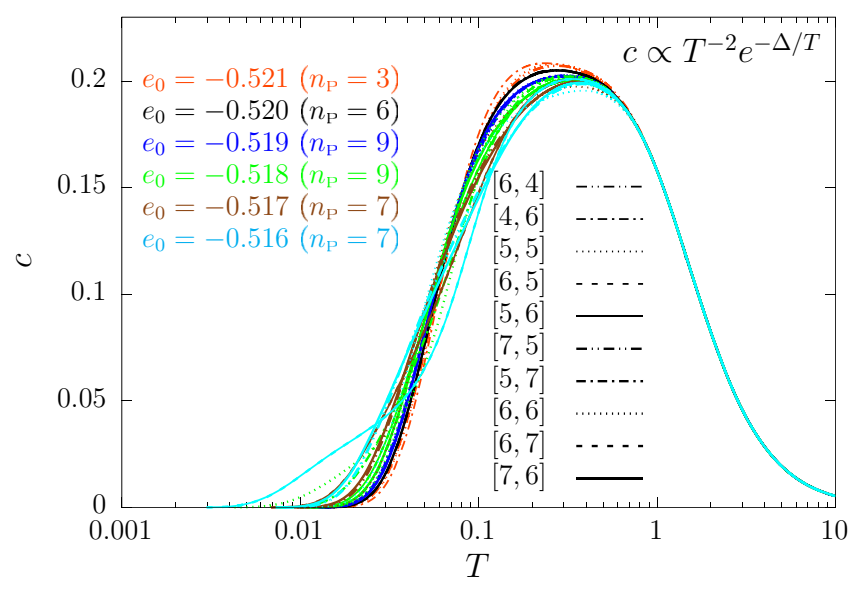

FIG. 7. EM results for the specific heat; gapped spectrum, $e_{0}=$ $-0.521 \ldots-0.516$. The parameter $n_{\mathrm{P}}$ in brackets behind energy values denotes the number of (very similar) Padé approximants shown here.

the results for $c(T)$ do not entirely discard a gapped ground state, further EM analysis of $\chi(T)$ under this assumption leads to disagreement with diagrammatic Monte Carlo simulations of Ref. [26] at $T<0.7$, see Fig. 13 below. We may consider these findings for $c(T)$ and $\chi(T)$ as an indication to favor a gapless ground state. We mention that the Green's function results indicate gapless magnetic excitations [25], and, as mentioned already above, the data for $\chi(T)$ given in Ref. [26] down to $T=J / 6$ seem also to be in favor of gapless excitations.

We focus now on the gapless case with a power-law decay of the specific heat $c(T)=A T^{\alpha}$ as $T \rightarrow 0$. Since we do not know the exponent $\alpha$, we study different values $\alpha=$ $1,3 / 2,2,5 / 2,3$. As for the gapped case, we again varied $e_{0}$ in the region $-0.57 \ldots-0.45$. We observed that only in a much smaller region around $e_{0}=-0.52$ reasonable results can be obtained (see also the discussion of Fig. 8, below). Thus, in what follows we consider preferably the region $-0.53 \ldots-0.50$ in more detail.

First we consider the prefactor $A_{\text {app }}$ that is given within the EM by Eq. (3.8). According to above outlined criteria for physically relevant EM outcomes the values of $A_{\text {app }}$ obtained by different Padé approximants must be very close to each other. From Fig. 8 (see also Fig. 2 in Ref. [70] for additional information) it is evident that for each value of $\alpha$ there is a well-defined relevant region of $e_{0}$, namely, $-0.515 \ldots-0.513$ for $\alpha=1,-0.518 \ldots-0.516$ for $\alpha=$ $3 / 2,-0.521 \ldots-0.518$ for $\alpha=2,-0.522 \ldots-0.520$ for $\alpha=5 / 2,-0.524 \ldots-0.522$ for $\alpha=3$. In all cases the ground-state energy is within the interval $e_{0}=-0.524 \ldots-$ 0.513 , which is much narrower than the wide region reported in the literature ranging from $e_{0}=-0.57$ to $e_{0}=$ $-0.45[12,13,15,16,22-25,69]$.

We consider the coincidence of $A$ values for different Padé approximants as a necessary criterion to figure out regions of relevant values for $e_{0}$ and $\alpha$. Since $A$ (together with $\alpha$ ) determines the $c(T)$ profile at sufficiently low $T$, we can get additional reliability by examining the region around the main ("high"-temperature) maximum. For that we compare

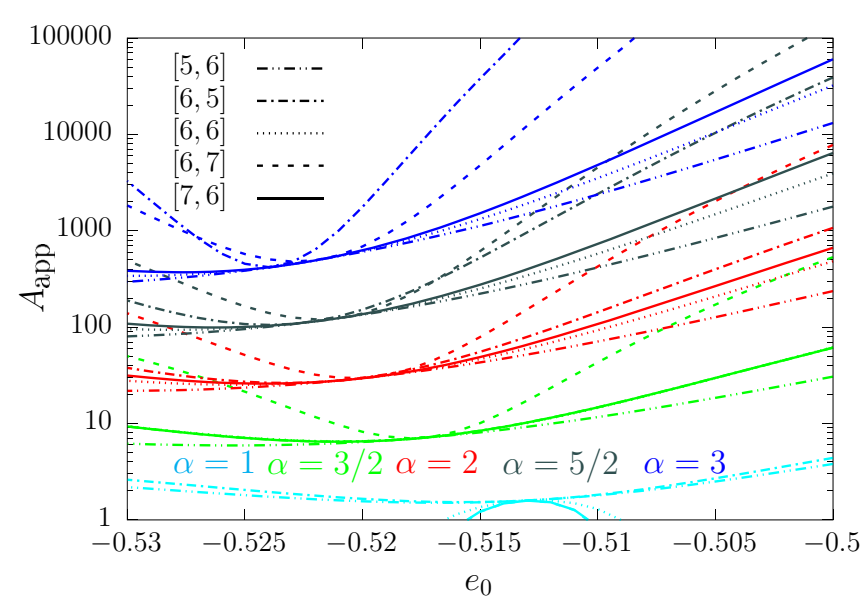

FIG. 8. EM for gapless spectrum: The prefactor $A_{\text {app }}$ as it follows from Eq. (3.8) for $\alpha=1,3 / 2,2,5 / 2,3$ versus the ground-state energy $e_{0}$ varied in the region $-0.53 \ldots-0.50$.

the position $T_{\max }$ and the height $c\left(T_{\max }\right)$ of this maximum obtained from different Padé approximants in dependence on $e_{0}$ within the regions guided by the previous inspection of $A$ for $\alpha=3 / 2,2,5 / 2$ in Fig. 9 (Fig. 3 in Ref. [70] reports such data for a wider region of $e_{0}$ including also $\alpha=1$ and 3). For each $\alpha$ we find pretty small regions of $e_{0}$ which yield almost identical $T_{\max }$ and $c\left(T_{\max }\right)$, and, consistently, this region fits well to the region obtained by inspection of $A$. For example, for $\alpha=2$ all Padé approximants give almost identical $T_{\max }$ and $c\left(T_{\max }\right)$, cf. Fig. 9 (red symbols), if $e_{0}$ is taken within the region $-0.522 \ldots-0.519$, which is in excellent agreement with that obtained from the prefactor $A$, see above. Note, however, that for $\alpha=1$ and 3 the diversity of $T_{\max }$ and $c\left(T_{\max }\right)$ is noticeably larger than for $\alpha=3 / 2,2,5 / 2$, cf. Fig. 3 in Ref. [70], indicating that the exponents $\alpha=1$ and 3 are less favorable.

Finally, after the specification of the ground-state energy values as outlined above, we present in Fig. 10 the full $c(T)$ curves obtained by the EM for $\alpha=3 / 2,2,5 / 2$ and a few related optimal values of $e_{0}$. [Corresponding curves for $\alpha=1$ and $\alpha=3$ are shown in Fig. 4 in Ref. [70]. Moreover, Fig. 5 in Ref. [70] provides additional results of $c(T)$ comparing data for all $\alpha=1,3 / 2,2,5 / 2,3$ for various values of $e_{0}$ ].

As can be seen in Fig. 10, there is a quite large number of Padé approximants (see the parameter $n_{\mathrm{P}}$ in brackets behind energy values) yielding very similar temperature profiles $c(T)$. Thus, for $\alpha=2$ we show in the middle panel of Fig. 10 $n_{\mathrm{P}}=10$ Padé approximants if $e_{0}=-0.519$ and -0.518 and $n_{\mathrm{P}}=9$ Padé approximants if $e_{0}=-0.521$ and $e_{0}=-0.520$. Outside this region of $e_{0}$ values the number of physical Padé approximants becomes noticeably smaller.

Without favoring any of the assumptions on the low- $T$ behavior of $c(T)$ and taking into account all (i.e., gapped and gapless excitations) EM predictions for $c(T)$ collected in Figs. 7 and 10 (see also Fig. 6 in Ref. [70], where we present a direct comparison of both cases), we have clear evidence (1) for a quite narrow region of reasonable $e_{0}$ values and (2) for the absence of a double-peak profile in $c(T)$. (3) Though, the position $T_{\max }$ and the height $c\left(T_{\max }\right)$ of the maximum of the specific heat slightly depend on the assumption about the 

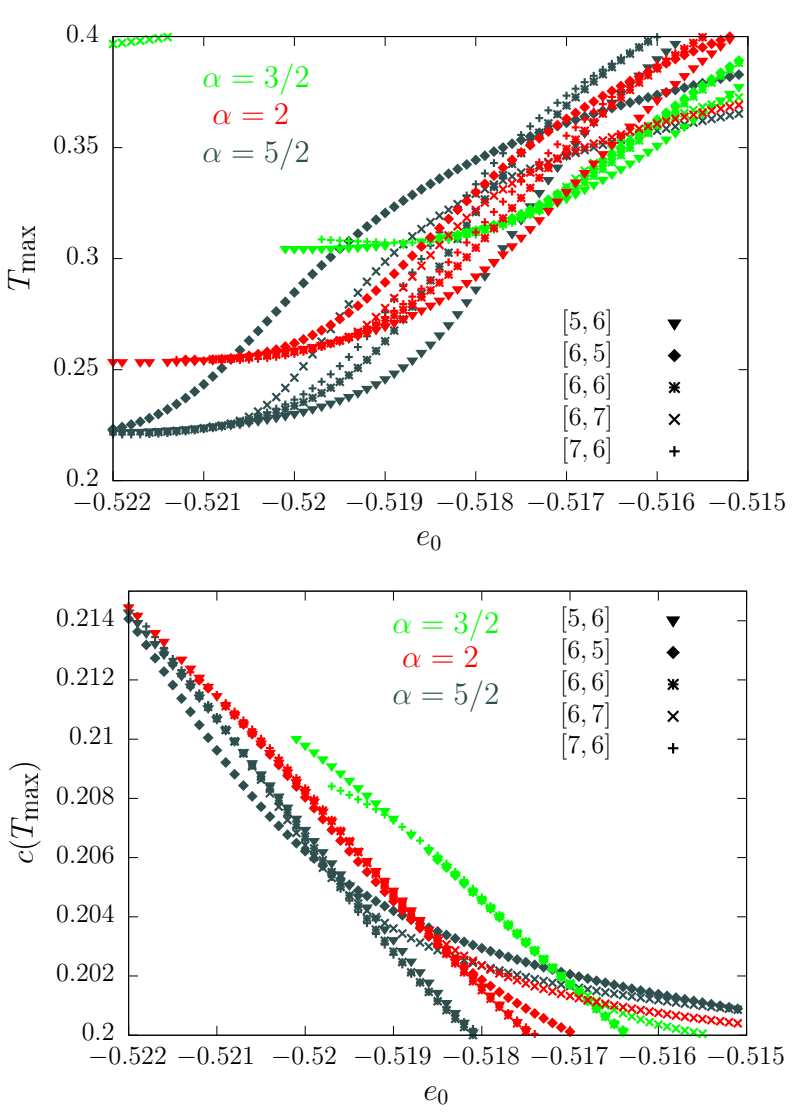

FIG. 9. EM for gapless spectrum: Position $T_{\max }$ (top) and height $c\left(T_{\max }\right)$ (bottom) of the specific heat versus the ground-state energy $e_{0}$ varied in the region region $-0.522 \ldots-0.515$ for exponents $\alpha=$ $3 / 2$ (green), $\alpha=2$ (red), and $\alpha=5 / 2$ (dark gray).

ground-state energy $e_{0}$ and low-lying excitations, all cases yield $T_{\max }$ around $\approx 0.25$ and $c\left(T_{\max }\right)$ around $\approx 0.2$.

Finally, we compare our EM results for $c(T)$ with data calculated by HTE (without subsequent EM interpolation) and by the Green's function approach [25], cf. Fig. 11. The Green's function results deviate from the EM results already below $T \sim 1$, whereas the HTE data deviate below $T \sim 0.7$.

The EM straightforwardly also provides the temperature profile of the entropy $s(T)$, see Fig. 12. Since the finitetemperature phase transition present in the simple-cubic HAF does not influence $s(T)$ as much as $c(T)$ [71], we compare the data for the PHAF with corresponding ones for the simplecubic HAF taken from Ref. [72]. We also show HTE data. Similar as already found for the finite system, cf. the middle panel of Fig. 3, the frustration leads to a much faster increase of $s$ at low temperatures for the PHAF. Thus, at $T=0.5$ the entropy already amounts to more than $50 \%$ of its maximal value $\ln 2 \approx 0.69$. (Note that in Fig. 7 in Ref. [70] we present a direct comparison of the gapped and gapless temperature profiles of $s$ ).

We consider now the static uniform susceptibility $\chi$ calculated by the EM as described in Sec. III A. According to the results of the rotation-invariant Green's function method, there is $\chi_{0} \equiv \chi(T=0)>0$. A nonzero $\chi_{0}$ may be also expected from the diagrammatic Monte Carlo simulations [26]. Nevertheless, we will not exclude from the beginning $\chi_{0}=$ 0 , i.e., a nonzero spin gap. Although the above discussed
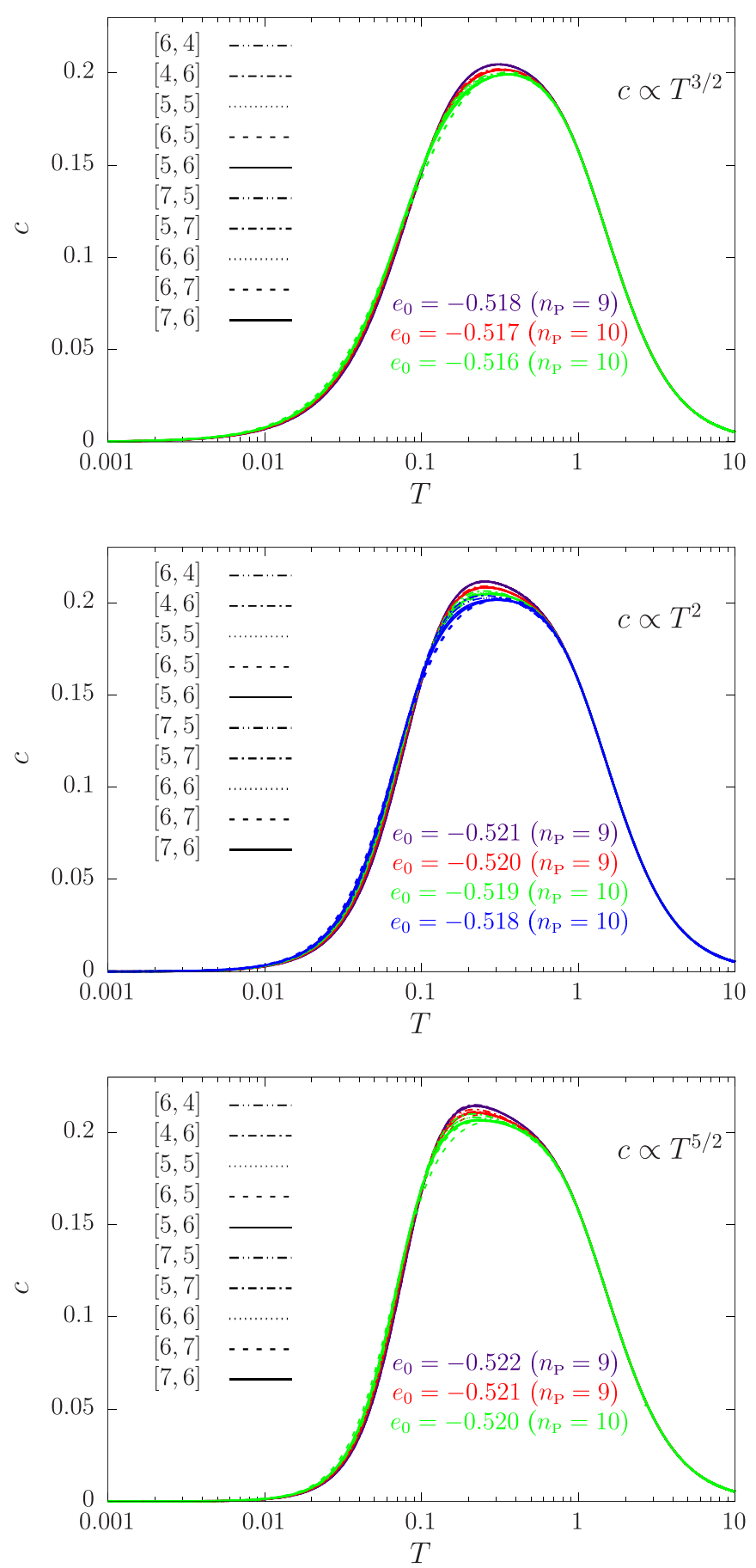

FIG. 10. EM results for the specific heat; gapless spectrum. (Top) $e_{0}=-0.518 \ldots-0.516, \alpha=3 / 2$. (Middle) $e_{0}=-0.521 \ldots-$ $0.518, \alpha=2$. (Bottom) $e_{0}=-0.522 \ldots-0.520, \alpha=5 / 2$. The parameter $n_{\mathrm{P}}$ in brackets behind energy values denotes the number of (very similar) Padé approximants shown here.

EM data for $c(T)$ are in favor of a gapless spectrum, the specific heat profiles were not fully conclusive to entirely reject the gapped spectrum. Moreover, one could have gapless singlet (i.e., nonmagnetic) excitations but gapped triplet (i.e., magnetic) excitations. Thus we studied the case with exponential decay of $\chi(T)$ and $c(T)$ as $T \rightarrow 0$ (i.e., gapped singlet and triplet excitations), see Fig. 13, as well as the case with exponential decay of $\chi(T)$ and power-law decay 


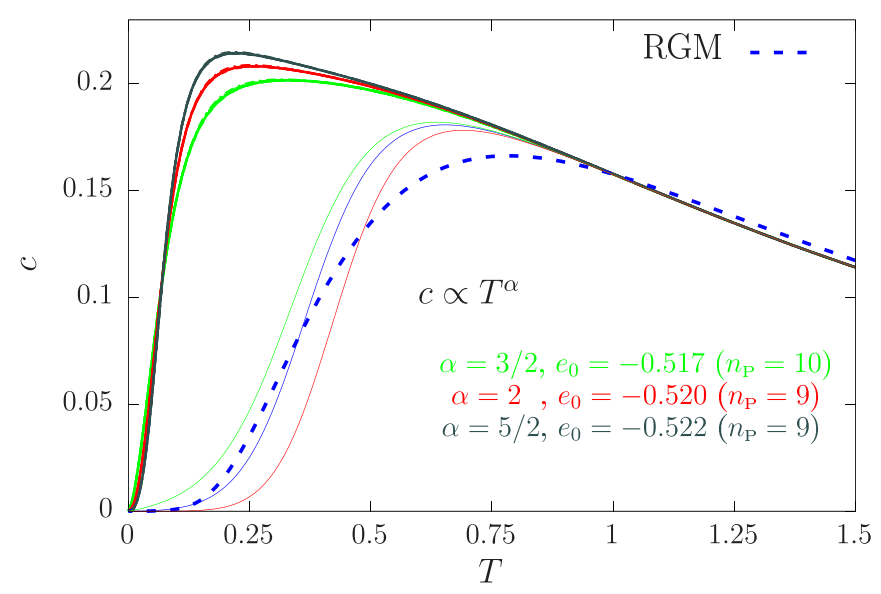

FIG. 11. Specific heat $c(T)$ of the $S=1 / 2$ PHAF: Comparison of our EM data $\left(e_{0}=-0.522, \alpha=5 / 2 ; e_{0}=-0.520, \alpha=2 ; e_{0}=\right.$ $-0.517, \alpha=3 / 2$ ) with results obtained by the rotation-invariant Green's function method [25] (dashed blue) and by the HTE (thin solid curves; we show the same HTE data for $N=\infty$ as shown in Fig. 4).

of $c(T)$ as $T \rightarrow 0$ (i.e., gapless singlet and gapped triplet excitations), see Fig. 14, where we consider those values for $e_{0}$ and $\alpha$ which are previously used to get $c(T)$ (see also Figs. 8 and 9 in Ref. [70]). From these figures, it is obvious that the susceptibility profiles based on gapped magnetic excitations are not compatible with the diagrammatic Monte Carlo data of Ref. [26] in the temperature region below 0.7, thus providing further evidence against a gapped spectrum. On the other hand, the EM results for gapless excitations with $\alpha=$ $3 / 2,2,5 / 2$ and $e_{0}=-0.522 \ldots-0.516$ and with nonzero $\chi_{0}$ as shown in Fig. 14 fit much better to the data of Ref. [26]. This argument complements the previous observations about the number of close Padé approximants for $c(T)$ [compare Figs. 7 (gapped) and 10 (gapless)] and for $\chi(T)$ [compare Figs. 13 (gapped) and 14 (gapless)]: The EM results favor the assumption of gapless excitations.

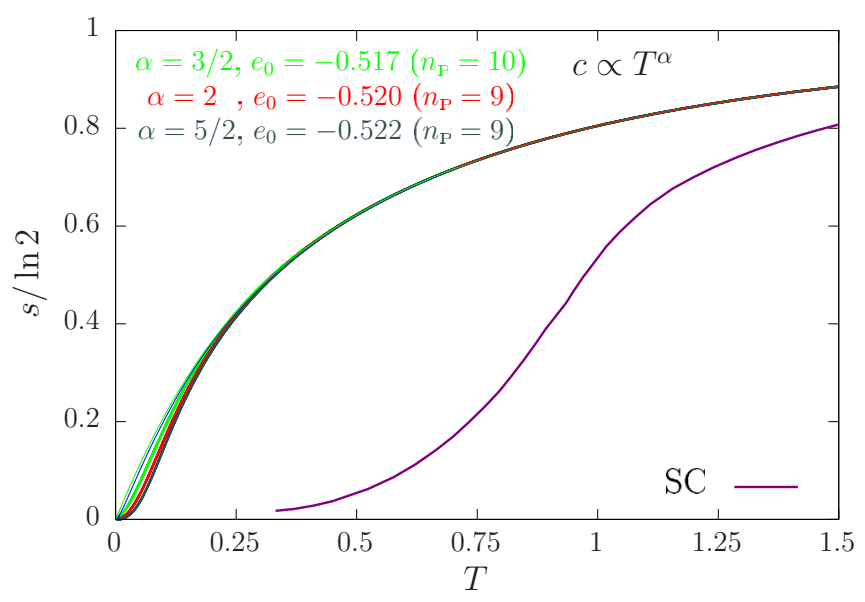

FIG. 12. EM data for the entropy for the gapless case with $e_{0}=$ $-0.522, \alpha=5 / 2 ; e_{0}=-0.520, \alpha=2 ; e_{0}=-0.517, \alpha=3 / 2$. We also show quantum Monte-Carlo data for the simple-cubic HAF [72] as well as HTE data (thin solid lines), where we show the same Padé approximants as used in Fig. 4 for $c(T)$. (Note that the HTE data are very close to the EM data).

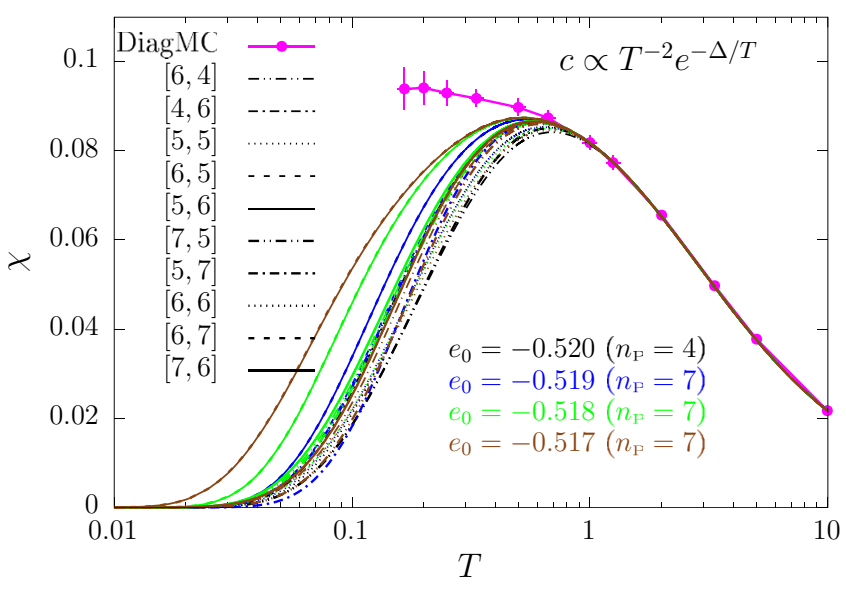

FIG. 13. EM results for the susceptibility; gapped spectrum, $e_{0}=-0.520 \ldots-0.517$.

As for the specific heat, we finally compare our EM results for $\chi(T)$ with data calculated by HTE (without subsequent EM interpolation), by the Green's function approach [25], cf. Fig. 15, where we also include the data of the diagrammatic Monte Carlo simulations [26]. The Green's function results deviate already below $T \sim 1.5$, and the HTE coincides down to $T \sim 0.7$, whereas $\chi(T)$ profiles with $\chi_{0}=0.1$ are in excellent agreement with the data of Ref. [26].

\section{CONCLUSIONS AND SUMMARY}

We have studied the specific heat $c(T)$, the entropy $s(T)$, and the static uniform susceptibility $\chi(T)$ of the spin-half pyrochlore Heisenberg antiferromagnet (PHAF) on a finite lattice of $N=32$ sites using the finite-temperature Lanczos method (FTLM) and on the infinite lattice using the hightemperature expansion (HTE) up to order 13 and a sophisticated interpolation between the low- and high-temperature behavior of the thermodynamic potential entropy $s$ as a function of internal energy $e$ [the entropy method (EM)].

We found that finite lattices of such size are not appropriate to get reasonable results below $T \sim 0.7$, but they might be useful to get a general impression on the strong frustration effects present in the PHAF by comparison of the HAF on finite pyrochlore and simple-cubic lattices. A similar limitation to temperatures above $T \sim 0.7$ is valid for the HTE even if the range of validity of the high-temperature series is extended by Padé approximants. Only the EM interpolation is suitable to overcome these limitations and to provide sound data for the whole temperature range.

Our main findings for the specific heat $c(T)$ are as follows. (i) Contrary to the two-dimensional kagome HAF, we do not find hints either for an extra low- $T$ peak or an extra shoulder below the main maximum. However, the absence of an extra low- $T$ feature goes hand in hand with a significant shift of the single maximum towards $T \sim 0.25$, which is much lower than for the kagome HAF, where the main maximum is at $T_{\max } / J=0.67$ [46,54]. This conclusion is robust, i.e., it is obtained not only for gapless excitations for all reasonable exponents $\alpha$ of the low-temperature power law of $c(T)$ but holds also for gapped excitations. (ii) A gapless spectrum is more favorable than a gapped one, i.e., most likely there is 

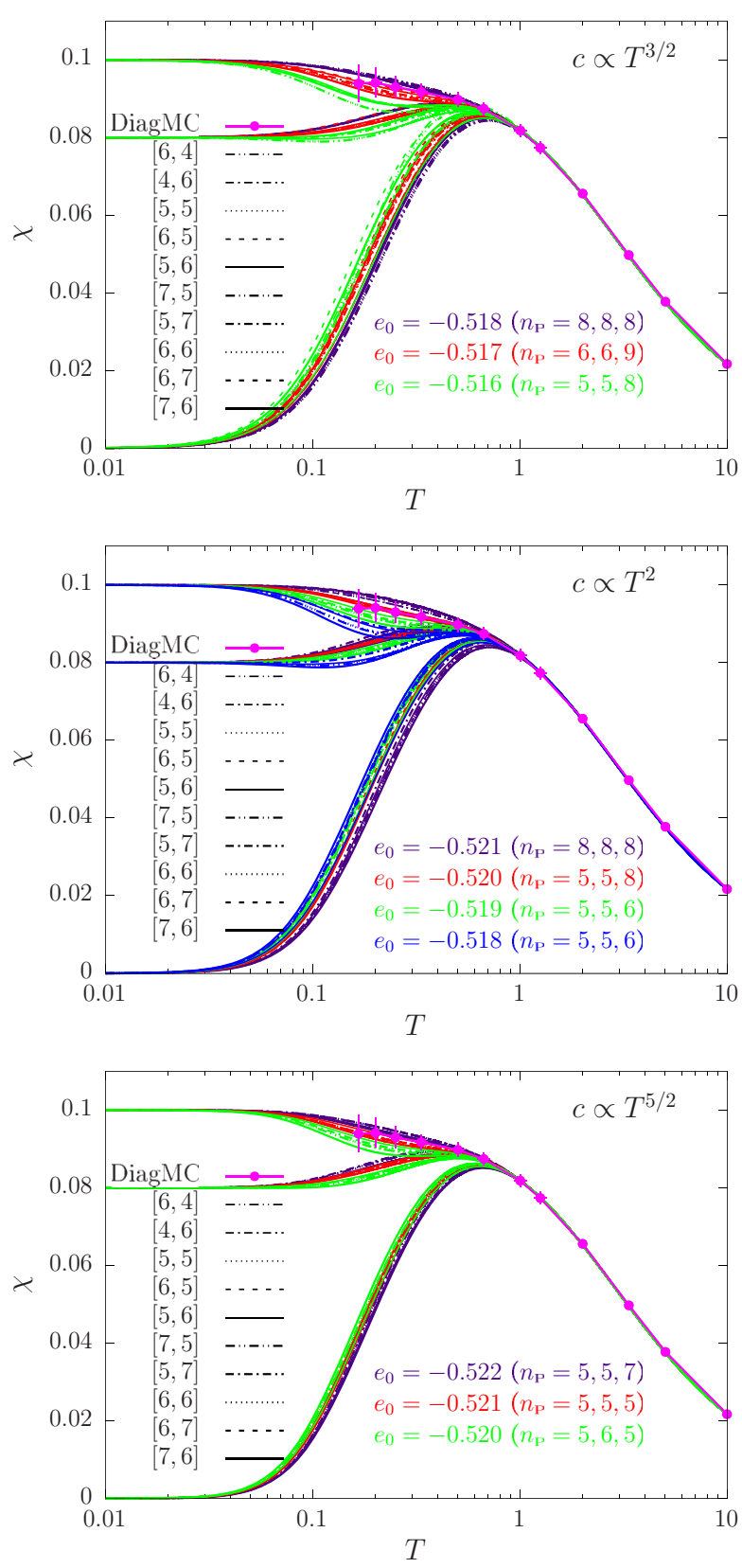

FIG. 14. EM results for the susceptibility; gapless spectrum. (Top) $e_{0}=-0.518 \ldots-0.516, \alpha=3 / 2$. (Middle) $e_{0}=$ $-0.521 \ldots-0.518, \alpha=2$. (Bottom) $e_{0}=-0.522 \ldots-0.520, \alpha=$ $5 / 2 . \chi_{0}=0,0.08,0.1$. Three values of $n_{\mathrm{P}}$ in brackets correspond to the assumed values $\chi_{0}=0.1, \chi_{0}=0.08$, and $\chi_{0}=0$, consequently.

power-law low- $T$ behavior of $c(T)$. Although best results are for an exponent $\alpha=2$, other exponents $(\alpha=1,3 / 2,5 / 2,3)$ cannot be excluded. (iii) We predict a ground-state energy $e_{0} \approx-0.52[73]$.

Our EM data for the susceptibility $\chi(T)$ in comparison with data obtained by diagrammatic Monte Carlo [26] provide further evidence for a gapless spectrum with a ground-state energy $e_{0} \approx-0.52$. The temperature profile of $\chi$ most likely does not show a maximum, rather there is a monotonous increase of $\chi$ upon decreasing of $T$ reaching a zero-temperature value of $\chi_{0} \approx 0.1$. Recently, we have learned that $R$. Schäfer et al. [74] also examined thermodynamics of the quantum

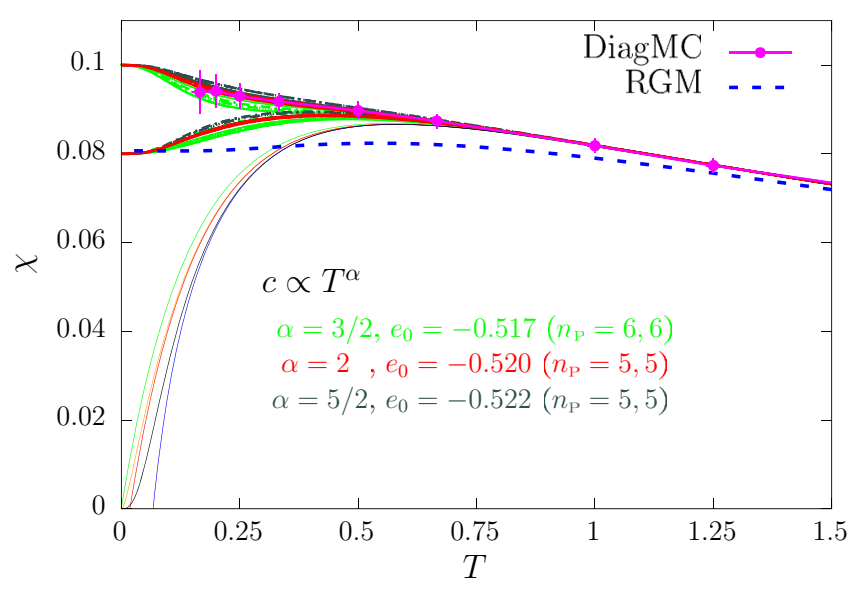

FIG. 15. Susceptibility $\chi(T)$ of the $S=1 / 2$ PHAF: Comparison of our EM data $\left(e_{0}=-0.522, \alpha=5 / 2 ; e_{0}=-0.520, \alpha=2 ; e_{0}=\right.$ $-0.517, \alpha=3 / 2$ ) with results obtained by the rotation-invariant Green's function method [25] (dashed blue), by the diagrammatic Monte Carlo [26], and by the HTE (thin solid curves; we show the same HTE data for $N=\infty$ as shown in Fig. 5).

PHAF, however, using for that numerical linked-cluster expansions.

\section{ACKNOWLEDGMENTS}

The authors gratefully acknowledge helpful discussions with David J. Luitz and Imre Hagymási. They thank Paul A. McClarty for his critical reading of the manuscript and helpful comments and suggestions and Michel J. P. Gingras for informing them about Refs. [31,32]. O.D. acknowledges the kind hospitality of the MPIPKS, Dresden in SeptemberNovember of 2019. T.H. was supported by the State Fund for Fundamental Research of Ukraine (Project No. F82/45950 "Effects of frustration in quantum spin systems") and by the fellowship of the National Academy of Sciences of Ukraine for young scientists.

\section{APPENDIX A: M. PLANCK's DERIVATION OF THE SPECIFIC HEAT $c(T)$ OF AN OSCILLATOR}

In his revolutionary paper in 1900 [28] (see also the Nobel Prize address [Notes 7, 12, and 13 at the end of the Nobel Prize address "The origin and development of the quantum theory" by Max Planck delivered before the Royal Swedish Academy of Sciences at Stockholm, 2 June, 1920] [42]), M. Planck investigated the energy distribution of electromagnetic radiation emitted by a black body in thermal equilibrium. For that he considered the entropy of the equilibrium radiation $S$ [75] in relation with its energy $U$, or more accurately, the second derivative $\mathrm{d}^{2} S / \mathrm{d} U^{2}$. According to Wien's law it is

$$
\frac{\mathrm{d}^{2} S}{\mathrm{~d} U^{2}}=-\frac{1}{b U} .
$$

But in view of experiments for high temperatures one has $U=$ $c T$, i.e.,

$$
\frac{\mathrm{d}^{2} S}{\mathrm{~d} U^{2}}=-\frac{c}{U^{2}}
$$


(M. Planck here refers to experiments by F. Kurlbaum.) While Wien's law (A1) is valid for small energy values (short wavelength), Eq. (A2) describes the high-energy limit (long wavelength, Rayleigh-Jeans law). To get agreement with experimental data $\mathrm{M}$. Planck suggested

$$
\frac{\mathrm{d}^{2} S}{\mathrm{~d} U^{2}}=-\frac{c}{U(b c+U)} \longrightarrow \begin{cases}-\frac{1}{b U}, & U \ll b c, \\ -\frac{c}{U^{2}}, & U \gg b c,\end{cases}
$$

which interpolates between both limiting cases. By integrating we get

$$
\frac{1}{T}=\frac{\mathrm{d} S}{\mathrm{~d} U}=\frac{1}{b} \ln \left(1+\frac{b c}{U}\right),
$$

which yields Planck's formula

$$
U=\frac{b c}{e^{b / T}-1} .
$$

These arguments can be formulated within the setup of the entropy method to find the specific heat $c(T)$ of a (Bose) oscillator, which represents the electromagnetic radiation with the frequency $v=2 \pi \omega$. Now we know that $b=\hbar \omega$ and $c=1$. Taking into account the zero-point energy we have to replace $U \rightarrow e=U+\hbar \omega / 2$. Then the Planck's interpolation formula (A3) for the auxiliary function $G(e)=(s(e))^{\prime \prime}$ reads:

$$
\begin{aligned}
G(e) & =-\frac{1}{\left(e-\frac{\hbar \omega}{2}\right)\left(e+\frac{\hbar \omega}{2}\right)}=\frac{1}{\left(\frac{\hbar \omega}{2}\right)^{2}-e^{2}} \\
& \longrightarrow\left\{\begin{array}{cl}
-\frac{1}{\hbar \omega\left(e-\frac{\hbar \omega}{2}\right)}, & e \ll \hbar \omega, \\
-\frac{1}{e^{2}}, & e \gg \hbar \omega .
\end{array}\right.
\end{aligned}
$$

The subscript app in the left-hand side of this equation is omitted since the suggested expression for $G(e)=(s(e))^{\prime \prime}$ (A6) appears to be exact. By integrating we get

$$
\frac{1}{T}=s^{\prime}(e)=\int_{e}^{\infty} \mathrm{d} e \frac{1}{e^{2}-\left(\frac{\hbar \omega}{2}\right)^{2}}=\frac{1}{\frac{\hbar \omega}{2}} \operatorname{arccth} \frac{e}{\frac{\hbar \omega}{2}}
$$

and then

$$
c(e)=-\frac{\left(s^{\prime}(e)\right)^{2}}{s^{\prime \prime}(e)}=\frac{e^{2}-\left(\frac{\hbar \omega}{2}\right)^{2}}{\left(\frac{\hbar \omega}{2}\right)^{2}} \operatorname{arccth}^{2} \frac{e}{\frac{\hbar \omega}{2}}
$$

and finally

$$
c(T)=\left(\frac{\frac{\hbar \omega}{2 T}}{\operatorname{sh} \frac{\hbar \omega}{2 T}}\right)^{2} .
$$

\section{APPENDIX B: HTE FOR THE $S=1 / 2$ PHAF}

We report here the HTE coefficients for the spin-half PHAF up to order 13 obtained by the Magdeburg HTE code developed mainly by A. Lohmann [59]. Note that the coefficients up to order 10 where presented previously for arbitrary spin $S$ in Refs. [27,60].

For the specific heat (per site) we have

$$
\begin{aligned}
c(\beta) & =\sum_{i} d_{i} \beta^{i}, \\
d_{1} & =0, \frac{d_{2}}{J^{2}}=+\frac{9}{16}, \quad \frac{d_{3}}{J^{3}}=-\frac{9}{32}, \quad \frac{d_{4}}{J^{4}}=-\frac{207}{256}, \\
\frac{d_{5}}{J^{5}} & =+\frac{195}{256}, \quad \frac{d_{6}}{J^{6}}=+\frac{3549}{4096}, \\
\frac{d_{7}}{J^{7}} & =-\frac{59073}{40960}, \quad \frac{d_{8}}{J^{8}}=-\frac{34535}{65536}, \\
\frac{d_{9}}{J^{9}} & =+\frac{345491}{163840}, \quad \frac{d_{10}}{J^{10}}=-\frac{9385203}{36700160}, \\
\frac{d_{11}}{J^{11}} & =-\frac{337285883}{132120576}, \quad \frac{d_{12}}{J^{12}}=+\frac{39036781051}{26424115200}, \\
\frac{d_{13}}{J^{13}} & =+\frac{144963365443}{58133053440} .
\end{aligned}
$$

For the static uniform susceptibility (per site) we have

$$
\begin{aligned}
\chi(\beta) & =\sum_{i} c_{i} \beta^{i}, \\
c_{1} & =+\frac{1}{4}, \quad \frac{c_{2}}{J}=-\frac{3}{8}, \quad \frac{c_{3}}{J^{2}}=+\frac{3}{8}, \quad \frac{c_{4}}{J^{3}}=-\frac{17}{64}, \\
\frac{c_{5}}{J^{4}} & =+\frac{85}{512}, \quad \frac{c_{6}}{J^{5}}=-\frac{97}{640}, \\
\frac{c_{7}}{J^{6}} & =+\frac{20207}{122880}, \quad \frac{c_{8}}{J^{7}}=-\frac{210989}{1720320}, \\
\frac{c_{9}}{J^{8}} & =+\frac{92147}{1966080}, \quad \frac{c_{10}}{J^{9}}=-\frac{4936709}{247726080}, \\
\frac{c_{11}}{J^{10}} & =+\frac{540939383}{9909043200}, \quad \frac{c_{12}}{J^{11}}=-\frac{5315724257}{72666316800}, \\
\frac{c_{13}}{J^{12}} & =+\frac{20479483351}{747424972800} .
\end{aligned}
$$

[1] J. S. Gardner, M. J. P. Gingras, and J. E. Greedan, Magnetic pyrochlore oxides, Rev. Mod. Phys. 82, 53 (2010).

[2] M. J. P. Gingras and P. A. McClarty, Quantum spin ice: A search for gapless quantum spin liquids in pyrochlore magnets, Rep. Prog. Phys. 77, 056501 (2014).

[3] J. G. Rau and M. J. P. Gingras, Frustrated quantum rareearth pyrochlores, Annu. Rev. Condens. Matter Phys. 10, 357 (2019).

[4] J. N. Reimers, A. J. Berlinsky, and A.-C. Shi, Mean-field approach to magnetic ordering in highly frustrated pyrochlores, Phys. Rev. B 43, 865 (1991).
[5] J. N. Reimers, Absence of long-range order in a threedimensional geometrically frustrated antiferromagnet, Phys. Rev. B 45, 7287 (1992).

[6] R. Moessner and J. T. Chalker, Properties of a Classical Spin Liquid: The Heisenberg Pyrochlore Antiferromagnet, Phys. Rev. Lett. 80, 2929 (1998).

[7] R. Moessner and J. T. Chalker, Low-temperature properties of classical geometrically frustrated antiferromagnets, Phys. Rev. B 58, 12049 (1998).

[8] S. V. Isakov, K. Gregor, R. Moessner, and S. L. Sondhi, Dipolar Spin Correlations in Classical Pyrochlore Magnets, Phys. Rev. Lett. 93, 167204 (2004). 
[9] C. L. Henley, The Coulomb phase in frustrated systems, Annu. Rev. Condens. Matter Phys. 1, 179 (2010).

[10] M. F. Lapa and C. L. Henley, Ground states of the classical antiferromagnet on the pyrochlore lattice, arXiv:1210.6810.

[11] Y. Iqbal, T. Müller, P. Ghosh, M. J. P. Gingras, H. O. Jeschke, S. Rachel, J. Reuther, and R. Thomale, Quantum and Classical Phases of the Pyrochlore Heisenberg Model with Competing Interactions, Phys. Rev. X 9, 011005 (2019).

[12] A. B. Harris, A. J. Berlinsky, and C. Bruder, Ordering by quantum fluctuations in a strongly frustrated Heisenberg antiferromagnet, J. Appl. Phys. 69, 5200 (1991).

[13] M. Isoda and S. Mori, Valence-bond crystal and anisotropic excitation spectrum on 3-dimensionally frustrated pyrochlore, J. Phys. Soc. Jpn. 67, 4022 (1998).

[14] B. Canals and C. Lacroix, Pyrochlore Antiferromagnet: A Three-Dimensional Quantum Spin Liquid, Phys. Rev. Lett. 80, 2933 (1998).

[15] B. Canals and C. Lacroix, Quantum spin liquid: The Heisenberg antiferromagnet on the three-dimensional pyrochlore lattice, Phys. Rev. B 61, 1149 (2000).

[16] A. Koga and N. Kawakami, Frustrated Heisenberg antiferromagnet on the pyrochlore lattice, Phys. Rev. B 63, 144432 (2001)

[17] H. Tsunetsugu, Antiferromagnetic quantum spins on the pyrochlore lattice, J. Phys. Soc. Jpn. 70, 640 (2001).

[18] H. Tsunetsugu, Spin-singlet order in a pyrochlore antiferromagnet, Phys. Rev. B 65, 024415 (2001).

[19] E. Berg, E. Altman, and A. Auerbach, Singlet Excitations in Pyrochlore: A Study of Quantum Frustration, Phys. Rev. Lett. 90, 147204 (2003).

[20] R. Moessner, S. L. Sondhi, and M. O. Goerbig, Quantum dimer models and effective Hamiltonians on the pyrochlore lattice, Phys. Rev. B 73, 094430 (2006).

[21] O. Tchernyshyov, R. Moessner, and S. L. Sondhi, Flux expulsion and greedy bosons: Frustrated magnets at large $N$, Europhys. Lett. 73, 278 (2006).

[22] J. H. Kim and J. H. Han, Chiral spin states in the pyrochlore Heisenberg magnet: Fermionic mean-field theory and variational Monte Carlo calculations, Phys. Rev. B 78, 180410(R) (2008).

[23] F. J. Burnell, S. Chakravarty, and S. L. Sondhi, Monopole flux state on the pyrochlore lattice, Phys. Rev. B 79, 144432 (2009).

[24] V. R. Chandra and J. Sahoo, Spin-1/2 Heisenberg antiferromagnet on the pyrochlore lattice: An exact diagonalization study, Phys. Rev. B 97, 144407 (2018).

[25] P. Müller, A. Lohmann, J. Richter, and O. Derzhko, Thermodynamics of the pyrochlore-lattice quantum Heisenberg antiferromagnet, Phys. Rev. B 100, 024424 (2019).

[26] Y. Huang, K. Chen, Y. Deng, N. Prokof'ev, and B. Svistunov, Spin-Ice State of the Quantum Heisenberg Antiferromagnet on the Pyrochlore Lattice, Phys. Rev. Lett. 116, 177203 (2016).

[27] H.-J. Schmidt, A. Lohmann, and J. Richter, Eighth-order hightemperature expansion for general Heisenberg Hamiltonians, Phys. Rev. B 84, 104443 (2011).

[28] M. Planck, Über eine Verbesserung der Wienschen Spektralgleichung, Verhandl. Dtsch. phys. Ges. 2, 202 (1900).

[29] B. Bernu and G. Misguich, Specific heat and high-temperature series of lattice models: Interpolation scheme and examples on quantum spin systems in one and two dimensions, Phys. Rev. B 63, 134409 (2001).
[30] G. Misguich and B. Bernu, Specific heat of the $s=\frac{1}{2}$ Heisenberg model on the kagome lattice: High-temperature series expansion analysis, Phys. Rev. B 71, 014417 (2005).

[31] L. Clark, G. J. Nilsen, E. Kermarrec, G. Ehlers, K. S. Knight, A. Harrison, J. P. Attfield, and B. D. Gaulin, From Spin Glass to Quantum Spin Liquid Ground States in Molybdate Pyrochlores, Phys. Rev. Lett. 113, 117201 (2014).

[32] Y. Iqbal, T. Müller, K. Riedl, J. Reuther, S. Rachel, R. Valentí, M. J. P. Gingras, R. Thomale, and H. O. Jeschke, Signatures of a gearwheel quantum spin liquid in a spin- $\frac{1}{2}$ pyrochlore molybdate Heisenberg antiferromagnet, Phys. Rev. Mater. 1, 071201(R) (2017).

[33] K. W. Plumb, H. J. Changlani, A. Scheie, S. Zhang, J. W. Kriza, J. A. Rodriguez-Rivera, Y. Qiu, B. Winn, R. J. Cava, and C. L. Broholm, Continuum of quantum fluctuations in a three-dimensional $S=1$ Heisenberg magnet, Nat. Phys. 15, 54 (2019).

[34] S. Zhang, H. J. Changlani, K. W. Plumb, O. Tchernyshyov, and R. Moessner, Dynamical Structure Factor of the ThreeDimensional Quantum Spin Liquid Candidate $\mathrm{NaCaNi}_{2} \mathrm{~F}_{7}$, Phys. Rev. Lett. 122, 167203 (2019).

[35] J. E. Greedan, M. Sato, X. Yan, and F. S. Razavi, Spin-glasslike behavior in $\mathrm{Y}_{2} \mathrm{Mo}_{2} \mathrm{O}_{7}$, a concentrated, crystalline system with negligible apparent disorder, Solid State Commun. 59, 895 (1986).

[36] H. J. Silverstein, K. Fritsch, F. Flicker, A. M. Hallas, J. S. Gardner, Y. Qiu, G. Ehlers, A. T. Savici, Z. Yamani, K. A. Ross, B. D. Gaulin, M. J. P. Gingras, J. A. M. Paddison, K. Foyevtsova, R. Valenti, F. Hawthorne, C. R. Wiebe, and $\mathrm{H}$. D. Zhou, Liquidlike correlations in single-crystalline $\mathrm{Y}_{2} \mathrm{Mo}_{2} \mathrm{O}_{7}$ : An unconventional spin glass, Phys. Rev. B 89, 054433 (2014).

[37] P. M. M. Thygesen, J. A. M. Paddison, R. Zhang, K. A. Beyer, K. W. Chapman, H. Y. Playford, M. G. Tucker, D. A. Keen, M. A. Hayward, and A. L. Goodwin, Orbital Dimer Model for the Spin-Glass State in $\mathrm{Y}_{2} \mathrm{Mo}_{2} \mathrm{O}_{7}$, Phys. Rev. Lett. 118, 067201 (2017).

[38] S. Gao, K. Guratinder, U. Stuhr, J. S. White, M. Mansson, B. Roessli, T. Fennell, V. Tsurkan, A. Loidl, M. Ciomaga Hatnean, G. Balakrishnan, S. Raymond, L. Chapon, V. O. Garlea, A. T. Savici, A. Cervellino, A. Bombardi, D. Chernyshov, Ch. Rüegg, J. T. Haraldsen, and O. Zaharko, Manifolds of magnetic ordered states and excitations in the almost Heisenberg pyrochlore antiferromagnet $\mathrm{MgCr}_{2} \mathrm{O}_{4}$, Phys. Rev. B 97, 134430 (2018).

[39] S. Ji, S.-H. Lee, C. Broholm, T. Y. Koo, W. Ratcliff, S.W. Cheong, and P. Zschack, Spin-Lattice Order in Frustrated $\mathrm{ZnCr}_{2} \mathrm{O}_{4}$, Phys. Rev. Lett. 103, 037201 (2009).

[40] M. Matsuda, M. Takeda, M. Nakamura, K. Kakurai, A. Oosawa, E. Lelièvre-Berna, J.-H. Chung, H. Ueda, H. Takagi, and S.-H. Lee, Spiral spin structure in the Heisenberg pyrochlore magnet $\mathrm{CdCr}_{2} \mathrm{O}_{4}$, Phys. Rev. B 75, 104415 (2007).

[41] A. Sadeghi, M. Alaei, F. Shahbazi, and M. J. P. Gingras, Spin Hamiltonian, order out of a Coulomb phase, and pseudocriticality in the frustrated pyrochlore Heisenberg antiferromagnet $\mathrm{FeF}_{3}$, Phys. Rev. B 91, 140407(R) (2015).

[42] M. Planck, The Origin of Quantum Theory (Nobel Prize Address 1920) (The Clarendon Press, Oxford, 1922).

[43] B. Bernu, C. Lhuillier, E. Kermarrec, F. Bert, P. Mendels, R. H. Colman, and A. S. Wills, Exchange energies of kapellasite from 
high-temperature series analysis of the kagome lattice $J_{1}-J_{2}-$ $J_{d}$-Heisenberg model, Phys. Rev. B 87, 155107 (2013).

[44] B. Bernu and C. Lhuillier, Spin Susceptibility of Quantum Magnets from High to Low Temperatures, Phys. Rev. Lett. 114, 057201 (2015).

[45] H.-J. Schmidt, A. Hauser, A. Lohmann, and J. Richter, Interpolation between low and high temperatures of the specific heat for spin systems, Phys. Rev. E 95, 042110 (2017).

[46] B. Bernu, L. Pierre, K. Essafi, and L. Messio, Effect of perturbations on the kagome $S=1 / 2$ antiferromagnet at all temperatures, Phys. Rev. B 101, 140403(R) (2020).

[47] J. Jaklič and P. Prelovšek, Lanczos method for the calculation of finite-temperature quantities in correlated systems, Phys. Rev. B 49, 5065 (1994).

[48] J. Jaklič and P. Prelovšek, Finite-temperature properties of doped antiferromagnets, Adv. Phys. 49, 1 (2000).

[49] J. Schnack and O. Wendland, Properties of highly frustrated magnetic molecules studied by the finite-temperature Lanczos method, Eur. Phys. J. B 78, 535 (2010).

[50] P. Prelovšek and J. Bonča, Ground state and finite temperature Lanczos methods, in Strongly Correlated Systems: Numerical Methods, edited by A. Avella and F. Mancini (Springer, Berlin, 2013).

[51] O. Hanebaum and J. Schnack, Advanced finite-temperature Lanczos method for anisotropic spin systems, Eur. Phys. J. B 87, 194 (2014).

[52] B. Schmidt and P. Thalmeier, Frustrated two dimensional quantum magnets, Phys. Rep. 703, 1 (2017).

[53] P. Prelovšek, The finite temperature Lanczos method and its applications, in The Physics of Correlated Insulators, Metals, and Superconductors, edited by E. Pavarini, E. Koch, R. Scalettar, and R. M. Martin (Forschungszentrum Jülich, Jülich, 2017).

[54] J. Schnack, J. Schulenburg, and J. Richter, Magnetism of the $N=42$ kagome lattice antiferromagnet, Phys. Rev. B 98, 094423 (2018).

[55] J. Schnack, J. Richter, and R. Steinigeweg, Accuracy of the finite-temperature Lanczos method compared to simple typicality-based estimates, Phys. Rev. Res. 2, 013186 (2020).

[56] J. Schulenburg, spinpack 2.56, Magdeburg University (2017), https://www-e.ovgu.de/jschulen/spin/.

[57] J. Richter and J. Schulenburg, The spin-1/2 $J_{1}-J_{2}$ Heisenberg antiferromagnet on the square lattice: Exact diagonalization for $N=40$ spins, Eur. Phys. J. B 73, 117 (2010).

[58] J. Oitmaa, C. Hamer, and W. Zheng, Series Expansion Methods for Strongly Interacting Lattice Models (Cambridge University Press, Cambridge, 2006).

[59] A. Lohmann, Diploma thesis, University Magdeburg, 2012.

[60] A. Lohmann, H.-J. Schmidt, and J. Richter, Tenth-order hightemperature expansion for the susceptibility and the specific heat of spin-s Heisenberg models with arbitrary exchange patterns: Application to pyrochlore and kagome magnets, Phys. Rev. B 89, 014415 (2014).

[61] http://www.uni-magdeburg.de/jschulen/HTE/.

[62] N. Elstner and A. P. Young, Spin-1/2 Heisenberg antiferromagnet on the kagome lattice: High-temperature expansion and exact-diagonalization studies, Phys. Rev. B 50, 6871 (1994).

[63] T. Nakamura and S. Miyashita, Thermodynamic properties of the quantum Heisenberg antiferromagnet on the kagomé lattice, Phys. Rev. B 52, 9174 (1995).

[64] P. Tomczak and J. Richter, Thermodynamical properties of the Heisenberg antiferromagnet on the kagomé lattice, Phys. Rev. B 54, 9004 (1996).

[65] T. Munehisa, An improved finite temperature Lanczos method and its application to the spin-1/2 Heisenberg model on the kagome lattice, World J. Condens. Matter Phys. 4, 134 (2014).

[66] T. Shimokawa and H. Kawamura, Finite-temperature crossover phenomenon in the $S=1 / 2$ antiferromagnetic Heisenberg model on the kagome lattice, J. Phys. Soc. Jpn. 85, 113702 (2016).

[67] X. Chen, S.-J. Ran, T. Liu, C. Peng, Y.-Z. Huang, and G. $\mathrm{Su}$, Thermodynamics of spin-1/2 kagome Heisenberg antiferromagnet: algebraic paramagnetic liquid and finite-temperature phase diagram, Science Bulletin 63, 1545 (2018).

[68] C. Hotta and K. Asano, Magnetic susceptibility of quantum spin systems calculated by sine square deformation: Onedimensional, square lattice, and kagome lattice Heisenberg antiferromagnets, Phys. Rev. B 98, 140405(R) (2018).

[69] R. R. Sobral and C. Lacroix, Order by disorder in the pyrochlore antiferromagnets, Solid State Commun. 103, 407 (1997).

[70] See Supplemental Material at http://link.aps.org/supplemental/ 10.1103/PhysRevB.101.174426 for more figures presenting EM data for the specific heat, the entropy, and the susceptibility which are briefly discussed but not shown as figures in Sec. IV B.

[71] The application of the EM interpolation scheme to the simplecubic HAF, which exhibits a phase transition to the lowtemperature Néel-ordered phase at $T_{c} \approx 0.946$ [A. W. Sandvik, Critical Temperature and the Transition from Quantum to Classical Order Parameter Fluctuations in the Three-Dimensional Heisenberg Antiferromagnet, Phys. Rev. Lett. 80, 5196 (1998)], as well as to other systems with finite-temperature phase transitions (for an example see Ref. [44]) is still an open question. Obviously, for such cases the dependence $s(e)$ is more complicated and simple auxiliary functions like those given in Eqs. (3.5) or (3.9) may not be sufficient to calculate thermodynamic quantities in the whole temperature range.

[72] S. Wessel, Critical entropy of quantum Heisenberg magnets on simple-cubic lattices, Phys. Rev. B 81, 052405 (2010).

[73] It is worth it to make the following remark here. As explained in the main text, we followed the protocol to determine the best ground-state energy suggested in Ref. [46], see Sec. III E in Supplemental Material of Ref. [46]. The authors of that paper tested this protocol and commented on its accuracy that is important, in particular, when the HTE is known at not very high orders.

[74] R. Schäfer, I. Hagymási, R. Moessner, and D. J. Luitz, The pyrochlore $S=1 / 2$ Heisenberg antiferromagnet at finite temperature, arXiv:2003.04898.

[75] In Eqs. (A1)-(A5) we use notations of Ref. [42]. 